\title{
Impact of examined lymph node count on prognosis in patients with lymph node-negative pancreatic body/tail ductal adenocarcinoma
}

\author{
Yu-Feng Li ${ }^{1,2}$, Yu-Cheng Xiang ${ }^{1,2}$, Qiu-Qiang Zhang ${ }^{1,2}$, Wei-Lin Wang ${ }^{1,2,3,4}$ \\ ${ }^{1}$ Department of Hepatobiliary and Pancreatic Surgery, The Second Affiliated Hospital, Zhejiang University School of Medicine, Hangzhou, China; \\ ${ }^{2}$ Key Laboratory of Precision Diagnosis and Treatment for Hepatobiliary and Pancreatic Tumor of Zhejiang Province, Hangzhou, China; ${ }^{3}$ Clinical \\ Medicine Innovation Center of Precision Diagnosis and Treatment for Hepatobiliary and Pancreatic Disease of Zhejiang University, Hangzhou, \\ China; ${ }^{4}$ Clinical Research Center of Hepatobiliary and Pancreatic Diseases of Zhejiang Province, Hangzhou, China \\ Contributions: (I) Conception and design: YF Li, WL Wang; (II) Administrative support: WL Wang; (III) Provision of study materials or patients: \\ YF Li; (IV) Collection and assembly of data: YF Li, YC Xiang, QQ Zhang; (V) Data analysis and interpretation: YF Li, YC Xiang; (VI) Manuscript \\ writing: All authors; (VII) Final approval of manuscript: All authors. \\ Correspondence to: Dr. Wei-Lin Wang. Department of Hepatobiliary and Pancreatic Surgery, The Second Affiliated Hospital, Zhejiang University \\ School of Medicine, No. 88 Jiefang Road, Hangzhou, China. Email: wam@zju.edu.cn.
}

Background: Because the overall prognosis remains dismal for patients with resected pancreatic cancer (PC), we aimed to explore the prognostic impact of examined lymph node (ELN) count on lymph node (LN)-negative pancreatic body/tail ductal adenocarcinoma.

Methods: Patients' data were extracted from the Surveillance, Epidemiology, and End Results (SEER) database (National Cancer Institute, USA) to investigate the relationship between ELN count and survival outcomes of LN-negative pancreatic body/tail ductal adenocarcinoma.

Results: A total of 700 patients were included, and the median number of ELNs was 11. The respective 1-, 3-, 5-year overall survival (OS) rates were 75.3\%, 37.7\%, 30.3\%, and the 1-, 3-, 5-year cancer-specific survival (CSS) were $78.3 \%, 41.7 \%, 34.5 \%$. The X-tile analysis showed that 14 was the most optimal cutoff for both OS and CSS. Kaplan-Meier survival analysis indicated that patients with ELNs $>14$ had better OS and CSS than ELNs $\leq 14$. Multivariate Cox analysis showed ELNs $\leq 14$ was an independent risk factor for both OS [hazard ratio (HR), 1.357; 95\% confidence interval (CI), 1.080-1.704; P=0.009] and CSS (HR, 1.394; 95\% CI, 1.092-1.778; $\mathrm{P}=0.008)$.

Conclusions: ELN count is associated with the survival rate in patients with LN-negative pancreatic body/tail ductal adenocarcinoma. Accurate nodal staging for these patients requires more than 14 ELNs.

Keywords: Lymph nodes (LNs); pancreatic cancer (PC); prognosis; Surveillance, Epidemiology, and End Results database (SEER database)

Submitted Mar 21, 2020. Accepted for publication Jul 14, 2020.

doi: $10.21037 /$ jgo-20-158

View this article at: http://dx.doi.org/10.21037/jgo-20-158

\section{Introduction}

Pancreatic cancer (PC) is one of the most lethal malignancies and is the seventh leading cause of cancerrelated death worldwide, with approximately 432,242 related deaths in $2018(1,2)$. So far, surgical resection remains the only potential curative treatment strategy, but the overall prognosis remains dismal for patients with resected PC, with an estimated 5-year overall survival (OS) of approximately $20 \%$ (3).

Lymph node (LN) status is considered an important prognostic factor for resected PC patients. Those with LN metastases have poor survival and higher risks of disease 
recurrence (4). Therefore, sufficient lymphadenectomy may play an important role in improving the survival of resected PC patients. Recently, the number of examined lymph nodes (ELNs) was found to reflect survival after resection of PC; that is, patients with more ELNs had better prognosis $(5-11)$. However, the minimum number of ELN to be resected remains controversial and varies from 12 to 15 in different guidelines $(6,12,13)$. Moreover, the optimal number of ELNs to adequately stratify survival has not yet been established.

In the current literature, most of the data about the number of ELNs are limited to resection of the entire pancreas or the pancreatic head, and few studies have considered the relationship between ELN count and survival in patients with pancreatic body/tail ductal adenocarcinoma. Therefore, we performed a population-based retrospective analysis based on the Surveillance, Epidemiology, and End Results (SEER) database to investigate the prognostic value of ELN count for LN-negative pancreatic body/tail ductal adenocarcinoma. We present the following article in accordance with the STROBE reporting checklist (available at http://dx.doi.org/10.21037/jgo-20-158).

\section{Methods}

\section{Data source and patient selection}

The SEER database is a program of the National Cancer Institute that has been updated annually since 1973 and accounts for approximately $30 \%$ of the United States' population. The present study cohort was derived from the database using SEER*Stat software (version 8.3.6). Patients diagnosed between 2004 and 2015 who underwent surgery of the primary tumor were included. Records were retrieved according to the International Classification of Disease for Oncology (3rd edition) (ICD-O-3) code for the pancreas (C25.0-C25.9). Only patients with positive histological confirmation of primary pancreatic adenocarcinoma or infiltrating ductal carcinoma (ICD-O-3 histology/behavior code $8500 / 3$ or $8140 / 3$ ) located in the body/tail of the pancreas with pathologically negative LNs were involved. Patients were excluded if they had distant metastasis, unknown TNM information, or incomplete follow-up data. The study was conducted in accordance with the Declaration of Helsinki (as revised in 2013). Institutional review board approval and informed consent were not required because the SEER database is publicly available, and the patient information is anonymous.

\section{Study variables}

The following information was exacted from the SEER database: year at diagnosis, age at diagnosis, sex, race, marital status, histologic grade, TNM information, the number of ELNs, radiotherapy, chemotherapy, survival, and follow-up. Patients were reviewed and restaged according to the 8th edition of the American Joint Committee on Cancer staging system (13). ELN count was abstracted according to the total number of ELNs. Only patients with one or more LN examined were included. Patients with an unknown number of ELNs were also excluded. OS was defined as the time from diagnosis to death from any cause. Patients still alive were censored. Cancer-specific survival (CSS) was defined as the time from diagnosis to death from PC. Patients still alive or who died from other causes were censored. The last follow-up in the present study was on 31 December 2016.

\section{Statistical analysis}

$\mathrm{X}$-tile software (version 3.6.1) was used to determine the optimal cutoff number of ELNs (14). Survival curves were created using the Kaplan-Meier method, and differences between the curves were compared by the log-rank test. Cox proportional hazards regression models were used to assess potential risk factors for survival outcomes. Risk factors with a $\mathrm{P}$ value $<0.1$ in the univariable analysis were entered into the multivariable analysis. The hazard ratio (HR) and $95 \%$ confidence interval $(\mathrm{CI})$ were calculated. All $\mathrm{P}$ values were two-tailed, and $\mathrm{P}<0.05$ was considered statistically significant. All statistical analyses were performed using SPSS for Windows, version 19 (SPSS Inc., Chicago, IL, USA).

\section{Results}

\section{Baseline characteristics}

A total of 700 pancreatic body/tail ductal adenocarcinoma patients who underwent resection between 2004 and 2015 were enrolled in the present study cohort. Their clinicopathologic features are shown in Table 1. The median age at diagnosis was 67 [interquartile range (IQR): 60-75] years, and 363 (51.9\%) patients were female. The 
Table 1 Baseline clinicopathological features of patients with pancreatic body/tail ductal adenocarcinoma in the SEER database

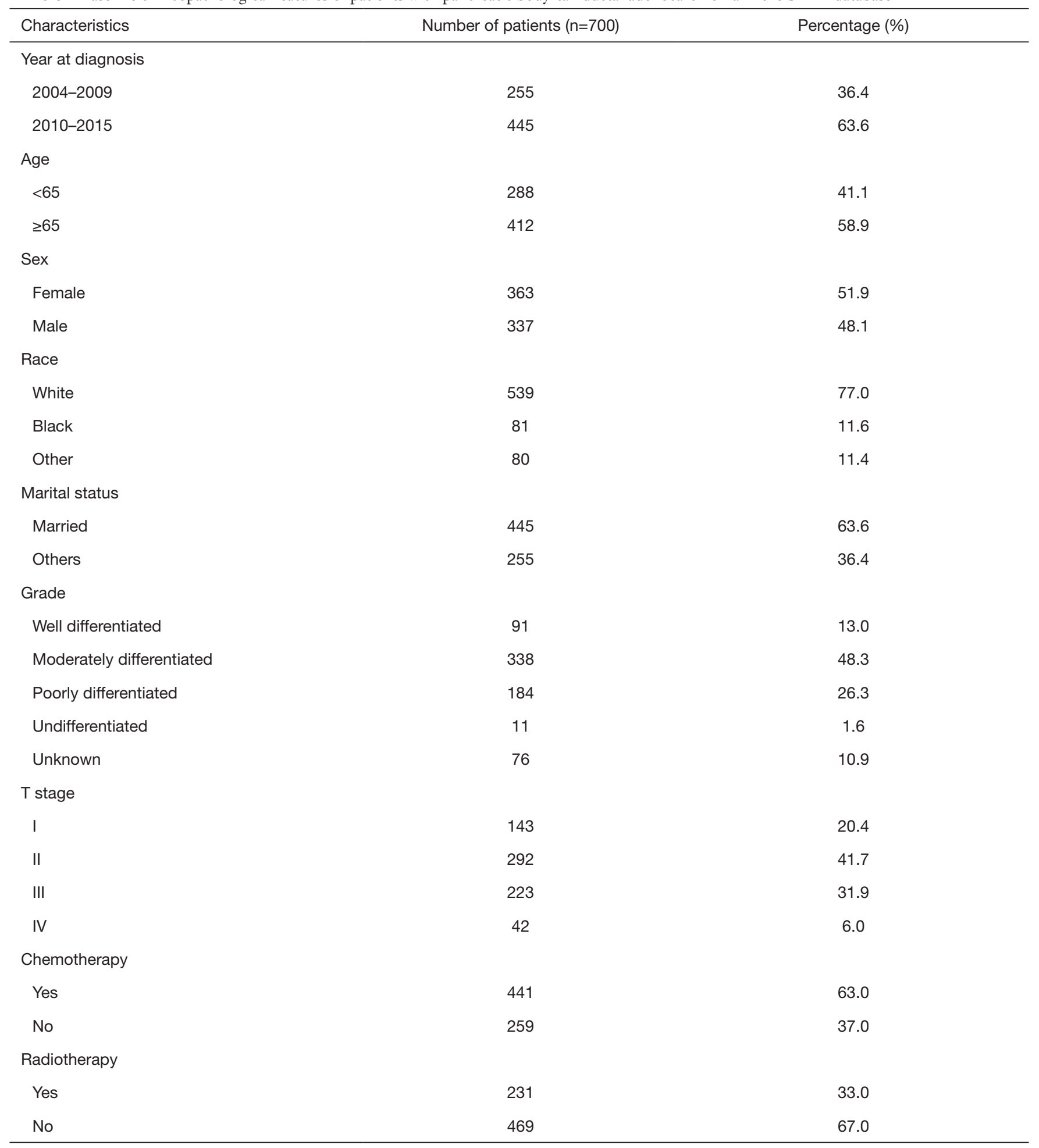

SEER, Surveillance, Epidemiology, and End Results. 
median number of ELNs was 11 (IQR: 5-16). The ELN distribution of the entire cohort is shown in Figure 1, and exact number of patients in ELN column is shown in Table S1.

\section{Survival analysis}

Of the 700 patients in the cohort, a total of 398 died during the follow-up period, comprising 352 deaths from PC and 46 deaths from other causes. The respective 1-, 3-, and 5 -year were $75.3 \%, 37.7 \%$, and $30.3 \%$ for OS, and $78.3 \%$, $41.7 \%$, and $34.5 \%$ for CSS. The Kaplan-Meier survival

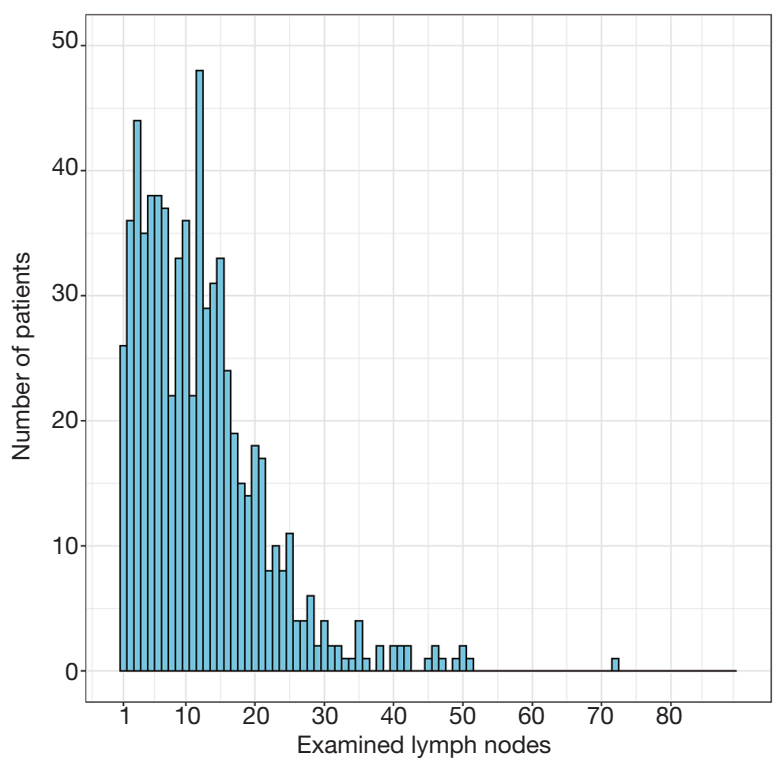

Figure 1 Distribution of ELNs in the entire cohort of patients with pancreatic body/tail ductal adenocarcinoma. ELN, examined lymph node. curves for OS and CSS are shown in Figure 2.

\section{Identification of the optimal cutoff value for ELN count}

$\mathrm{X}$-tile analysis to identify the cutoff for the optimal number of ELNs revealed 14 as the optimal cutoff for both OS and CSS (Figure S1). Kaplan-Meier survival analysis showed that patients with ELNs $>14$ had better OS and CSS than those with ELNs $\leq 14$ (Figure 3). Multivariate Cox analysis was performed after adjusting for available variables, and ELNs $\leq 14$ was found to be an independent risk factor for both OS (HR, 1.357; 95\% CI, 1.080-1.704; P=0.009) and CSS (HR, 1.394; 95\% CI, 1.092-1.778; $\mathrm{P}=0.008$ ) (Tables 2,3). Other independent prognostic factors identified in both the OS and CSS multivariate analyses included histologic grade, $\mathrm{T}$ stage, and receipt of chemotherapy.

\section{Discussion}

$\mathrm{LN}$ metastasis is regarded as an important prognostic factor in cancer survival. Cancer patients with $\mathrm{LN}$ metastasis have a poorer prognosis and higher risk of recurrence after surgical resection. Complete lymphadenectomy plays an important role in precise nodal staging and the appropriate delivery of adjuvant therapies. The number of ELNs is a well-known determinant factor for nodal staging. Previous studies have found that the number of ELNs has a close relationship with survival in PC (5-11). Patients with a higher number of ELNs might have better survival, especially in node-negative disease. Huebner $e t$ al. reported that pancreaticoduodenectomy patients with $>11$ ELNs had better OS compared with those with $<11$ ELNs (HR, 1.33, 95\% CI, 1.1-1.7; $\mathrm{P}=0.001$ ) (5). Slidell et al. reported that patients undergoing pancreatectomy for pancreatic adenocarcinoma should have at least $12 \mathrm{LNs}$ evaluated (7).
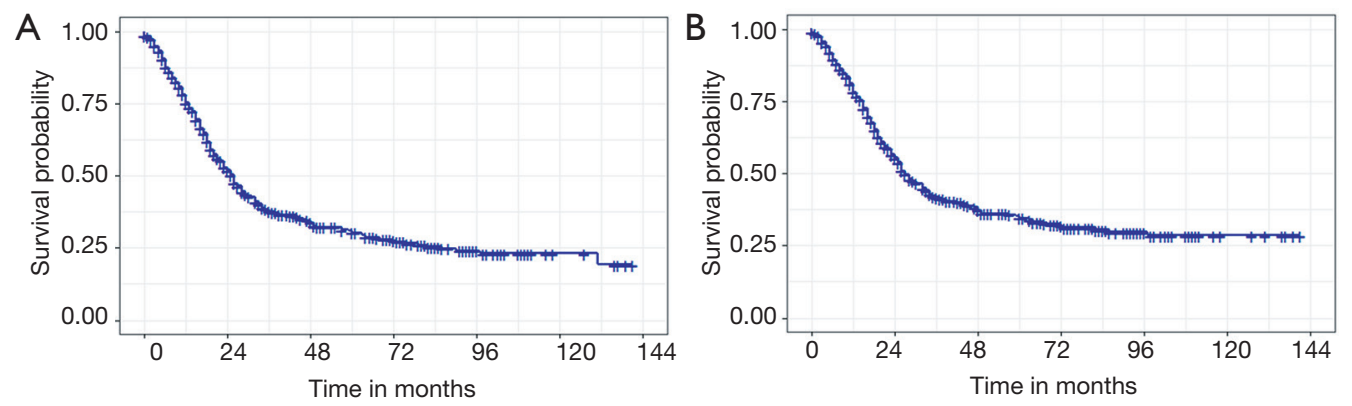

Figure 2 Kaplan-Meier curves of survival in patients with pancreatic body/tail ductal adenocarcinoma. (A) OS; (B) CSS. OS, overall survival; CSS, cancer-specific survival. 
A
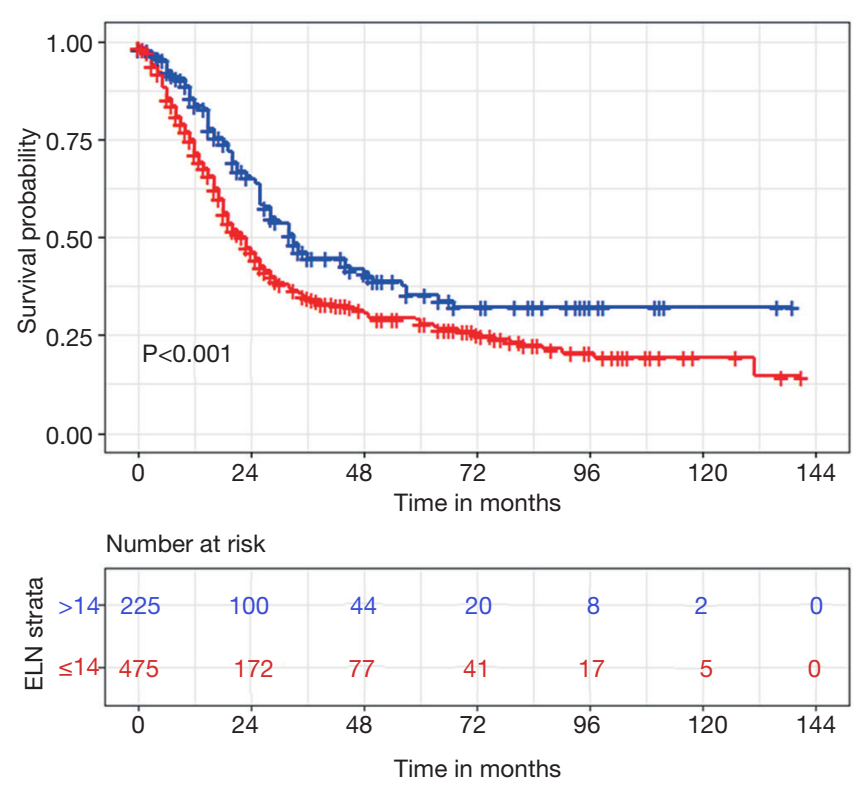

B
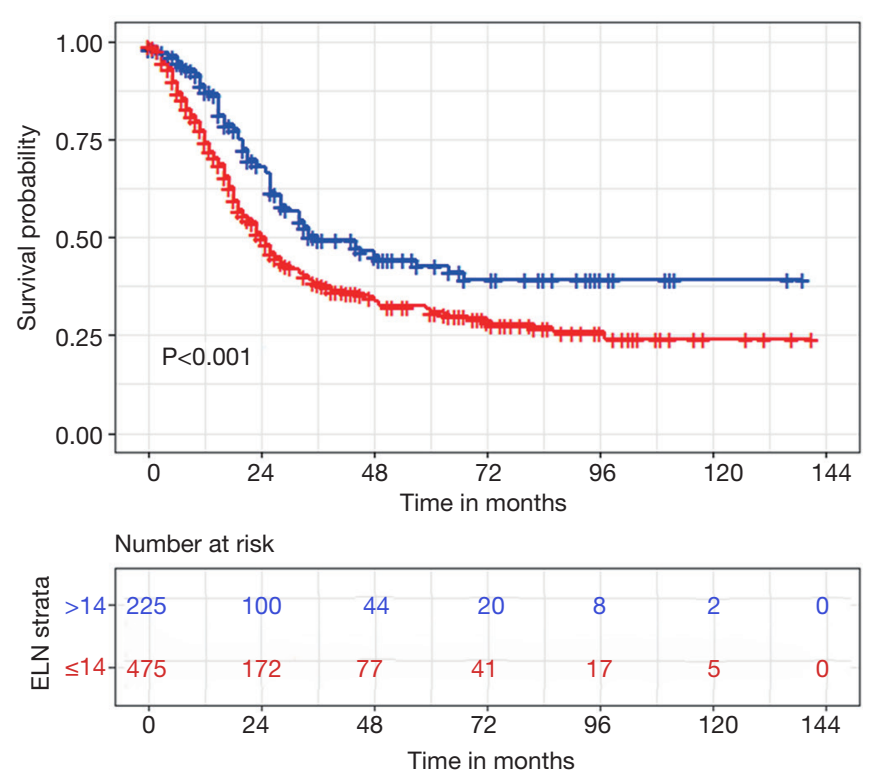

Figure 3 Kaplan-Meier curves of survival in patients with pancreatic body/tail ductal adenocarcinoma according to ELN count. (A) OS; (B) CSS. OS, overall survival; CSS, cancer-specific survival; ELN, examined lymph node.

A recent analysis from the Netherlands suggested 11 ELNs as the minimum and 19 ELNs as the optimal cutoff points for evaluating the quality of LN examination and possibly for stratifying postoperative prognosis (8). Based on these studies, the number of ELNs needed to be resected varies in different guidelines. The American Joint Committee on Cancer and Union for International Cancer Control recommends a minimum of $12 \mathrm{LNs}$ to be analyzed for nodal staging in PC (13). In contrast, the European Society for Medical Oncology recommends removal of at least $15 \mathrm{LNs}$ to obtain adequate staging (12). Moreover, the National Comprehensive Cancer Network has not suggested an optimal number of ELNs for PC.

Most PCs are located in the head of the pancreas, and only $20-25 \%$ in the body/tail (15). Tumor biology $(16,17)$, as well as surgical strategy, differs between the PC of the head and that of the body/tail. The resection of the head of the pancreas usually involves a pancreaticoduodenectomy, whereas resection of the body/tail involves a distal pancreatectomy $(18,19)$. However, for pancreatic body/ tail ductal adenocarcinoma, the optimal number of ELNs remains undetermined. Only two studies in the current literature have focused on this issue. Ashfaq et al. (9) found that 11 was the optimal ELN count for pancreatic adenocarcinoma patients undergoing distal pancreatectomy.
Patients with 1-10 ELNs had a worse survival than those with $>10$ ELNs. Another study conducted by Malleo et al. in a series of 240 patients with pancreatic body/tail ductal adenocarcinoma found the optimal ELN count was 20, and this was an independent prognostic factor in LN-negative patients (20).

In this study, we used the SEER database to explore the relationship between ELNs and survival in LN-negative pancreatic body/tail ductal adenocarcinoma. We found ELN was an independent prognostic factor for LNnegative pancreatic body/tail cancer patients. Moreover, we recommend that the number of ELNs should be $>14$ for these patients, which is compatible with the findings of previous studies.

The correlation of increased ELN count and improved survival can be explained by several potential reasons. First, a higher number of ELNs is associated with improved detection of positive LNs and more accurate staging. Patients with an inadequate number of ELNs and diagnosed node-negative may be node-positive. Second, more positive LNs will be detected with increasing ELN count, and a node-positive diagnosis will affect the delivery of adjuvant therapy after resection, which may improve survival. Third, more dissection of LNs may reflect the quality of surgery and pathological examination. A previous 
Table 2 Univariate and multivariate Cox proportional hazards regression analysis for OS

\begin{tabular}{|c|c|c|c|c|c|c|}
\hline Characteristics & \multicolumn{3}{|c|}{ Univariate analysis } & \multicolumn{3}{|c|}{ Multivariate analysis } \\
\hline \multicolumn{7}{|l|}{ Year at diagnosis } \\
\hline 2004-2009 & Reference & & & Reference & & \\
\hline 2010-2015 & 0.740 & $0.605-0.905$ & 0.003 & 0.872 & $0.708-1.074$ & 0.199 \\
\hline$<65$ & Reference & & & Reference & & \\
\hline$\geq 65$ & 1.368 & $1.116-1.677$ & 0.003 & 1.144 & $0.920-1.422$ & 0.227 \\
\hline \multicolumn{7}{|l|}{ Sex } \\
\hline Female & Reference & & & - & & \\
\hline White & Reference & & & Reference & & \\
\hline Black & 1.106 & $0.826-1.480$ & 0.499 & 1.037 & $0.760-1.415$ & 0.819 \\
\hline Other & 0.736 & $0.523-1.035$ & 0.078 & 0.825 & $0.584-1.165$ & 0.275 \\
\hline \multicolumn{7}{|l|}{ Marital status } \\
\hline Married & Reference & & & Reference & & \\
\hline Others & 1.237 & $1.011-1.513$ & 0.038 & 1.255 & $1.012-1.555$ & 0.039 \\
\hline \multicolumn{7}{|l|}{ Grade } \\
\hline Well/moderately differentiated & Reference & & & Reference & & \\
\hline III & 2.918 & $2.116-4.025$ & $<0.001$ & 2.951 & $2.116-4.117$ & $<0.001$ \\
\hline IV & 3.342 & $2.074-5.385$ & $<0.001$ & 4.517 & $2.763-7.383$ & $<0.001$ \\
\hline \multicolumn{7}{|l|}{ Chemotherapy } \\
\hline Yes & Reference & & & Reference & & \\
\hline No & 1.566 & $1.284-1.911$ & $<0.001$ & 1.814 & $1.470-2.239$ & $<0.001$ \\
\hline \multicolumn{7}{|l|}{ Radiotherapy } \\
\hline Yes & Reference & & & - & & \\
\hline No & 1.171 & $0.953-1.437$ & 0.133 & - & - & - \\
\hline \multicolumn{7}{|l|}{ ELN } \\
\hline$>14$ & Reference & & & Reference & & \\
\hline$\leq 14$ & 1.483 & $1.186-1.855$ & 0.001 & 1.357 & $1.080-1.704$ & 0.009 \\
\hline
\end{tabular}

OS, overall survival; HR, hazard ratio; Cl, confidence interval; ELN, examined lymph node. 
Table 3 Univariate and multivariate Cox proportional hazards regression analysis for CSS

\begin{tabular}{|c|c|c|c|c|c|c|}
\hline Characteristics & \multicolumn{3}{|c|}{ Univariate analysis } & \multicolumn{3}{|c|}{ Multivariate analysis } \\
\hline \multicolumn{7}{|l|}{ Year at diagnosis } \\
\hline 2004-2009 & Reference & & & Reference & & \\
\hline 2010-2015 & 0.705 & $0.570-0.873$ & 0.001 & 0.832 & $0.668-1.035$ & 0.099 \\
\hline$<65$ & Reference & & & Reference & & \\
\hline$\geq 65$ & 1.225 & $0.989-1.517$ & 0.063 & 1.040 & $0.833-1.300$ & 0.728 \\
\hline \multicolumn{7}{|l|}{ Sex } \\
\hline Female & Reference & & & - & & \\
\hline White & Reference & & & - & & \\
\hline Black & 1.059 & $0.773-1.452$ & 0.721 & - & - & - \\
\hline Other & 0.790 & $0.556-1.124$ & 0.190 & - & - & - \\
\hline \multicolumn{7}{|l|}{ Marital status } \\
\hline Married & Reference & & & - & & \\
\hline Others & 1.191 & $0.961-1.476$ & 0.111 & - & - & - \\
\hline \multicolumn{7}{|l|}{ Grade } \\
\hline Well/moderately differentiated & Reference & & & Reference & & \\
\hline III & 3.054 & $2.163-4.311$ & $<0.001$ & 3.121 & $2.187-4.455$ & $<0.001$ \\
\hline IV & 3.258 & $1.943-5.463$ & $<0.001$ & 3.980 & $2.348-6.746$ & $<0.001$ \\
\hline \multicolumn{7}{|l|}{ Chemotherapy } \\
\hline Yes & Reference & & & Reference & & \\
\hline No & 1.512 & $1.223-1.869$ & $<0.001$ & 1.798 & $1.437-2.249$ & $<0.001$ \\
\hline \multicolumn{7}{|l|}{ Radiotherapy } \\
\hline Yes & Reference & & & - & & \\
\hline No & 1.107 & $0.891-1.375$ & 0.359 & - & - & - \\
\hline \multicolumn{7}{|l|}{ ELN } \\
\hline$>14$ & Reference & & & Reference & & \\
\hline$\leq 14$ & 1.555 & $1.222-1.977$ & $<0.001$ & 1.394 & $1.092-1.778$ & 0.008 \\
\hline
\end{tabular}

CSS, cancer-specific survival; HR, hazard ratio; Cl, confidence interval; ELN, examined lymph node. 
study has indicated that high-volume medical centers have higher ELN counts and better survival (21). However, another key issue requires attention: the role of extended lymphadenectomy. The extent of lymphadenectomy needs to be considered against the risks of potential postoperative complications (3). Several previous studies have shown no survival difference between extended lymphadenectomy and standard lymphadenectomy (22-26). Therefore, more prospective studies need to focus on the extent of lymphadenectomy with the optimal number of ELNs.

There are several study limitations that should be mentioned. First, we could not avoid some bias because of the retrospective nature of the study. Second, the LNs removed and counted may have varied among different institutions in the SEER program. The number of ELNs depends on both the extent of the surgeon's lymphadenectomy and the pathologist's examination (27). Moreover, LN station and location could not be analyzed. Third, detailed information on surgery, margin status, postoperative complications, and recurrence status, as well as the receipt of systemic therapy, could not be assessed from the SEER database. We could not analyze the relationship between recurrence and ELN count. Moreover, for some patients (especially T4N0), surgery may not require the first standard treatment according to present guidelines, and they may have received neoadjuvant treatment or palliative surgical treatment, which could result in insufficient or absent nodal sampling and positive surgical margins, leading to inaccurate ELN count. Because of these inherent limitations, further prospective studies are required to confirm our findings.

\section{Conclusions}

Our study revealed that ELN count was an independent prognostic factor for patients with LN-negative pancreatic body/tail ductal adenocarcinoma. More than 14 ELNs are recommended for accurate nodal staging in these patients. Further prospective research is required to confirm our findings.

\section{Acknowledgments}

The authors thank the Surveillance, Epidemiology, and End Results Program for providing high-quality data.

Funding: This work was supported by the National Natural Science Foundation of China (Nos. 81572307 and 81773096) and the Major Project of Medical and Health
Technology Development Program in Zhejiang Province (No. 7211902).

\section{Footnote}

Reporting Checklist: The authors have completed the STROBE reporting checklist. Available at: http://dx.doi. org/10.21037/jgo-20-158

Conflicts of Interest: All authors have completed the ICMJE uniform disclosure form (available at http://dx.doi. org/10.21037/jgo-20-158). The authors have no conflicts of interest to declare.

Ethical Statement: The authors are accountable for all aspects of the work in ensuring that questions related to the accuracy or integrity of any part of the work are appropriately investigated and resolved. The study was conducted in accordance with the Declaration of Helsinki (as revised in 2013). Institutional review board approval and informed consent were not required because the SEER database is publicly available and the patient information is anonymous.

Open Access Statement: This is an Open Access article distributed in accordance with the Creative Commons Attribution-NonCommercial-NoDerivs 4.0 International License (CC BY-NC-ND 4.0), which permits the noncommercial replication and distribution of the article with the strict proviso that no changes or edits are made and the original work is properly cited (including links to both the formal publication through the relevant DOI and the license). See: https://creativecommons.org/licenses/by-nc-nd/4.0/.

\section{References}

1. Kamisawa T, Wood LD, Itoi T, et al. Pancreatic cancer. Lancet 2016;388:73-85.

2. Bray F, Ferlay J, Soerjomataram I, et al. Global cancer statistics 2018: GLOBOCAN estimates of incidence and mortality worldwide for 36 cancers in 185 countries. CA Cancer J Clin 2018;68:394-424.

3. Strobel O, Neoptolemos J, Jäger D, et al. Optimizing the outcomes of pancreatic cancer surgery. Nat Rev Clin Oncol 2019;16:11-26.

4. Khorana AA, Mangu PB, Berlin J, et al. Potentially curable pancreatic cancer: American Society of Clinical Oncology Clinical Practice Guideline. J Clin Oncol 
2016;34:2541-56.

5. Huebner M, Kendrick M, Reid-Lombardo KM, et al. Number of lymph nodes evaluated: prognostic value in pancreatic adenocarcinoma. J Gastrointest Surg 2012;16:920-6.

6. Tol JAMG, Gouma DJ, Bassi C, et al. Definition of a standard lymphadenectomy in surgery for pancreatic ductal adenocarcinoma: a consensus statement by the International Study Group on Pancreatic Surgery (ISGPS). Surgery 2014;156:591-600.

7. Slidell MB, Chang DC, Cameron JL, et al. Impact of total lymph node count and lymph node ratio on staging and survival after pancreatectomy for pancreatic adenocarcinoma: a large, population-based analysis. Ann Surg Oncol 2008;15:165-74.

8. Huang L, Jansen L, Balavarca Y, et al. Significance of examined lymph node number in accurate staging and long-term survival in resected stage I-II pancreatic cancermore is better? A large international population-based cohort study. Ann Surg 2019. [Epub ahead of print].

9. Ashfaq A, Pockaj BA, Gray RJ, et al. Nodal counts and lymph node ratio impact survival after distal pancreatectomy for pancreatic adenocarcinoma. J Gastrointest Surg 2014;18:1929-35.

10. Vuarnesson H, Lupinacci RM, Semoun O, et al. Number of examined lymph nodes and nodal status assessment in pancreaticoduodenectomy for pancreatic adenocarcinoma. Eur J Surg Oncol 2013;39:1116-21.

11. Warschkow R, Widmann B, Beutner U, et al. The more the better-lower rate of stage migration and better survival in patients with retrieval of 20 or more regional lymph nodes in pancreatic cancer. Pancreas 2017;46:648-57.

12. Ducreux M, Cuhna AS, Caramella C, et al. Cancer of the pancreas: ESMO Clinical Practice Guidelines for diagnosis, treatment and follow-up. Ann Oncol 2015;26 Suppl 5:v56-68.

13. Chun YS, Pawlik TM, Vauthey JN. 8th Edition of the AJCC Cancer Staging Manual: pancreas and hepatobiliary cancers. Ann Surg Oncol 2018;25:845-7.

14. Camp RL, Dolled-Filhart M, Rimm DL. X-tile: a new bio-informatics tool for biomarker assessment and outcome-based cut-point optimization. Clin Cancer Res 2004;10:7252-9.

15. Modolell I, Guarner L, Malagelada JR. Vagaries of clinical presentation of pancreatic and biliary tract cancer. Ann Oncol 1999;10 Suppl 4:82-4.

16. Birnbaum DJ, Bertucci F, Finetti P, et al. Head and body/ tail pancreatic carcinomas are not the same tumors. Cancers (Basel) 2019;11:497.

17. Dreyer SB, Jamieson NB, Upstill-Goddard R, et al. Defining the molecular pathology of pancreatic body and tail adenocarcinoma. Br J Surg 2018;105:e183-91.

18. Lau MK, Davila JA, Shaib YH. Incidence and survival of pancreatic head and body and tail cancers: a populationbased study in the United States. Pancreas 2010;39:458-62.

19. Tomasello G, Ghidini M, Costanzo A, et al. Outcome of head compared to body and tail pancreatic cancer: a systematic review and meta-analysis of 93 studies. J Gastrointest Oncol 2019;10:259-69.

20. Malleo G, Maggino L, Ferrone CR, et al. Number of examined lymph nodes and nodal status assessment in distal pancreatectomy for body/tail ductal adenocarcinoma. Ann Surg 2019;270:1138-46.

21. Lidsky ME, Sun Z, Nussbaum DP, et al. Going the extra mile: improved survival for pancreatic cancer patients traveling to high-volume centers. Ann Surg 2017;266:333-8.

22. Yeo CJ, Cameron JL, Sohn TA, et al. Pancreaticoduodenectomy with or without extended retroperitoneal lymphadenectomy for periampullary adenocarcinoma: comparison of morbidity and mortality and short-term outcome. Ann Surg 1999;229:613-22; discussion 622-4.

23. Pedrazzoli S, DiCarlo V, Dionigi R, et al. Standard versus extended lymphadenectomy associated with pancreatoduodenectomy in the surgical treatment of adenocarcinoma of the head of the pancreas: a multicenter, prospective, randomized study. Lymphadenectomy Study Group. Ann Surg 1998;228:508-17.

24. Yeo CJ, Cameron JL, Lillemoe KD, et al. Pancreaticoduodenectomy with or without distal gastrectomy and extended retroperitoneal lymphadenectomy for periampullary adenocarcinoma, part 2: randomized controlled trial evaluating survival, morbidity, and mortality. Ann Surg 2002;236:355-66; discussion 366-8.

25. Riall TS, Cameron JL, Lillemoe KD, et al. Pancreaticoduodenectomy with or without distal gastrectomy and extended retroperitoneal lymphadenectomy for periampullary adenocarcinoma-part 3: update on 5-year survival. J Gastrointest Surg 2005;9:1191-204; discussion 1204-6.

26. Sun J, Yang Y, Wang X, et al. Meta-analysis of the efficacies of extended and standard pancreatoduodenectomy for ductal adenocarcinoma of the head of the pancreas. World 
J Surg 2014;38:2708-15.

27. Adsay NV, Basturk O, Altinel D, et al. The number of lymph nodes identified in a simple pancreatoduodenectomy specimen: comparison of conventional vs orangepeeling approach in pathologic assessment. Mod Pathol 2009;22:107-12.

Cite this article as: Li YF, Xiang YC, Zhang QQ, Wang WL. Impact of examined lymph node count on prognosis in patients with lymph node-negative pancreatic body/tail ductal adenocarcinoma. J Gastrointest Oncol 2020;11(4):644-653. doi: 10.21037/jgo-20-158 


\section{Supplementary}

Table S1 ELN distribution of the entire cohort

\begin{tabular}{|c|c|c|}
\hline ELN counts & Patients number & Percentage (\%) \\
\hline 1 & 26 & 3.7 \\
\hline 2 & 36 & 5.1 \\
\hline 3 & 44 & 6.3 \\
\hline 4 & 35 & 5.0 \\
\hline 5 & 38 & 5.4 \\
\hline 6 & 38 & 5.4 \\
\hline 7 & 37 & 5.3 \\
\hline 8 & 22 & 3.1 \\
\hline 9 & 33 & 4.7 \\
\hline 10 & 36 & 5.1 \\
\hline 11 & 22 & 3.1 \\
\hline 12 & 48 & 6.9 \\
\hline 13 & 29 & 4.1 \\
\hline 14 & 31 & 4.4 \\
\hline 15 & 33 & 4.7 \\
\hline 16 & 24 & 3.4 \\
\hline 17 & 19 & 2.7 \\
\hline 18 & 15 & 2.1 \\
\hline 19 & 14 & 2.0 \\
\hline 20 & 18 & 2.6 \\
\hline 21 & 17 & 2.4 \\
\hline 22 & 8 & 1.1 \\
\hline 23 & 10 & 1.4 \\
\hline 24 & 8 & 1.1 \\
\hline 25 & 11 & 1.6 \\
\hline 26 & 4 & 0.6 \\
\hline 27 & 4 & 0.6 \\
\hline 28 & 6 & 0.9 \\
\hline 29 & 2 & 0.3 \\
\hline 30 & 4 & 0.6 \\
\hline 31 & 2 & 0.3 \\
\hline 32 & 2 & 0.3 \\
\hline 33 & 1 & 0.1 \\
\hline 34 & 1 & 0.1 \\
\hline 35 & 4 & 0.6 \\
\hline 36 & 1 & 0.1 \\
\hline 38 & 2 & 0.3 \\
\hline 40 & 2 & 0.3 \\
\hline 41 & 2 & 0.3 \\
\hline 42 & 2 & 0.3 \\
\hline 45 & 1 & 0.1 \\
\hline 46 & 2 & 0.3 \\
\hline 47 & 1 & 0.1 \\
\hline 49 & 1 & 0.1 \\
\hline 50 & 2 & 0.3 \\
\hline 51 & 1 & 0.1 \\
\hline 72 & 1 & 0.1 \\
\hline Total & 700 & 100.0 \\
\hline
\end{tabular}

ELN, examined lymph node. 


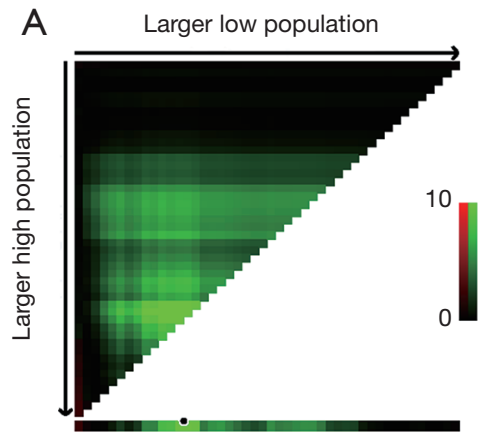

B

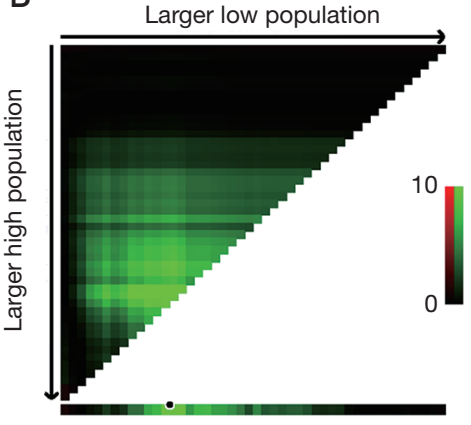

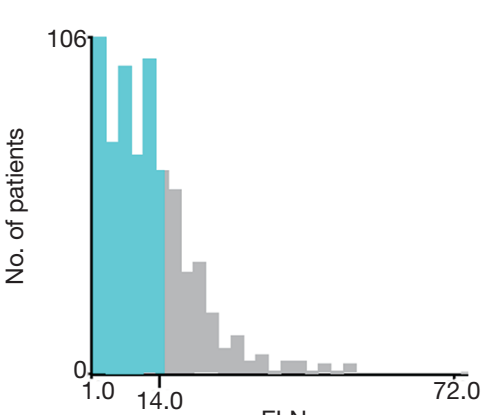

ELN

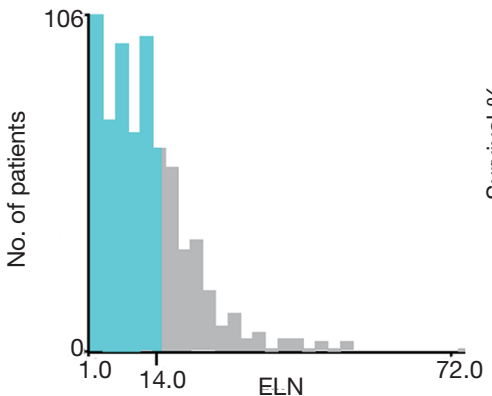

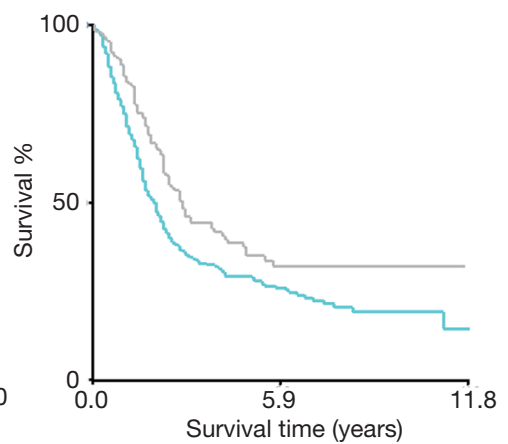

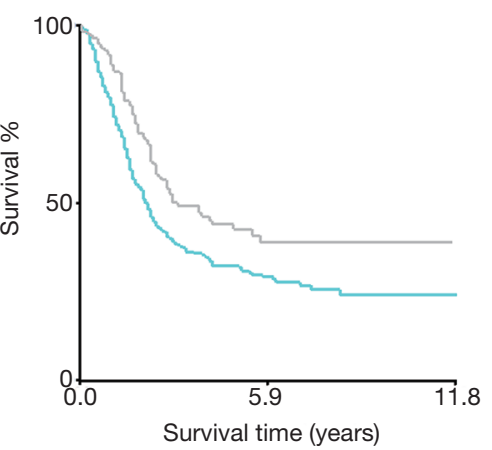

Figure S1 X-tile analysis of survival data in patients with pancreatic body/tail ductal adenocarcinoma according to ELN count. (A) OS; (B) CSS. OS, overall survival; CSS, cancer-specific survival; ELN, examined lymph node. 